\title{
ELECTRON DENSITY IN THE EMISSION-LINE REGION \\ OF WOLF-RAYET STARS
}

\author{
Y. P. VARSHNI \\ Dept of Physics, University of Ottawa, Ottawa, Canada
}

(Received 30 November, 1977; accepted 20 January, 1978)

\begin{abstract}
The Inglis-Teller relation, generalized for a hydrogen-like or alkali-like ion with an arbitrary core charge, is used to estimate the electron density in the emission-line region of WolfRayet stars. It is found that the electron density in the region which gives rise to He II emission lines is $\simeq 4 \times 10^{14} \mathrm{~cm}^{-3}$.
\end{abstract}

The electron density in the atmosphere of a star is a quantity of fundamental importance in determining the nature of the spectrum of the star. One method of determining this quantity is based on the relation due to Inglis and Teller (1939). The broadening of the Balmer lines by the static Stark effect increases with the principal quantum number $n$ until, at a certain quantum number, the Stark splitting is equal to the energy separation of the levels with adjacent principal quantum numbers. At this maximum value of the quantum number, say $n_{m}$, the spectral series ends and the energy levels of the atom merge with the continuum. There have been attempts (IvanovKholodnyi and Nikol'skii, 1961; Kurochka, 1965, 1967, 1974; Mitrofanov, 1973) to improve upon the Inglis-Teller relation. However, the Inglis-Teller formula is still a good representation of the experimental results (Nissen, 1954; Griem, 1964; Jefferies, 1968).

Varshni and Lam (1976) have presented evidence for the existence of laser action in the atmospheres of Wolf-Rayet stars (see also Varshni, 1977). In order to understand the excitation mechanism(s) of emission lines in these stars, it is obviously of importance to delineate the physical characteristics of the emission line region as closely as possible.

In this note we present a generalization of the Inglis-Teller relation for a hydrogenlike or alkali-like ion with an arbitrary charge of the core immersed in a plasma which is a mixture of ions of various charges and electrons. We use this relation to estimate the electron density in the emission-line region of the Wolf-Rayet stars.

Some thirty years ago, Aller (1943) made an estimate of this quantity, but he had to make a number of approximations to obtain his result. The present view regarding the magnitude of the electron density in the emission-line region of Wolf-Rayet stars is summarized by Underhill (1968) who states '.. it seems unlikely that the electron density exceeds $10^{14}$ in a typical Wolf-Rayet atmosphere'.

Edlén $(1931,1932,1933,1956)$ has, over the years, patiently and assiduously pursued the task of identification of the emission lines recorded in the spectra of

Astrophysics and Space Science 56 (1978) 385-388. All Rights Reserved

Copyright (C) 1978 by D. Reidel Publishing Company, Dordrecht, Holland 
Wolf-Rayet stars. An examination of the tables given in his 1956 paper shows that there is some evidence for transitions involving $n$ as high as 17 for $\mathrm{He}$ II in the spectrum of the WC 7 star HD 192103. It must be noted, however, that the lines identified as due to transitions 5-16 and 5-17 have 0 intensity. For $C$ IV the transitions with the highest $n$ value which are clearly identified have $n=15$. It is possible that transitions involving higher $n$ values, up to $n=21$, might be present, but these are either very faint features or are masked by other lines. The spectrum of HD 192103 has also been studied by Underhill (1959) and Bappu (1973). Underhill finds that the 4- $n$ and the $5-n$ series of He II may be followed to $n=21$. We may note here that the lines identified by her as due to 4-20 and 4-21 transitions have other contributors also. The line identified as 5-21 is very weak. Bappu (1973) states that the He II 5-n series can be followed up to the transition 5-21.

We consider a hydrogen-like or alkali-like atom or ion, the charge on the core that the valence electron experiences being equal to $Z e$, where $e$ is the electron charge. The maximum separation of the Stark components for the level having its principal quantum number equal to $n$ is (Bethe and Salpeter, 1957)

$$
E=3 F e a_{0} \frac{n(n-1)}{Z}
$$

where $F$ is the electric field and $a_{0}$ the Bohr radius. Following Inglis and Teller (1939), we equate it to the energy separation of the levels $n$ and $(n+1)$ to obtain

$$
3 F e a_{0} \frac{n(n-1)}{Z}=\frac{e^{2}}{2 a_{0}} Z^{2}\left[\frac{1}{n^{2}}-\frac{1}{(n+1)^{2}}\right] .
$$

Equation (2) determines the merger point, $n=n_{m}$, and can be approximately written as

$$
3 F e a_{0}=\frac{e^{2}}{a_{0}} \frac{Z^{3}}{n_{m}^{5}}
$$

The electric field due to the perturbers (in the surrounding plasma) based on the Holtsmark distribution is

$$
F=2.6\left|Z_{p} e\right| N_{p}^{2 / 3},
$$

where $\left|Z_{p} e\right|$ and $N_{p}$ are the charge and density $\left(\mathrm{cm}^{-3}\right)$, respectively, of the perturbing ions.

At low plasma temperatures, the electrons contribute to the broadening through the static Stark effect rather than through collisions. In this case the number of perturbers is taken to be the sum of the number of positive ions and electrons. At high temperatures, the positive ions alone cause the static Stark effect. It is customary to use the low-temperature approximation in the present context and we shall also do the same. The atom or ion which we are considering is immersed in the stellar plasma which consists of a mixture of atoms in various degrees of ionization and of 
free electrons. To simplify the picture we replace this plasma by an 'average' plasma which has $N_{i}$ number of positive ions per $\mathrm{cm}^{3}$ with an average charge $\zeta e$. On the average there will also be $\zeta$ free electrons per positive ion $\left(\right.$ per $\mathrm{cm}^{3}$ ). The total density of perturbers is given by

$$
N_{p}=N_{i}+\zeta N_{i}
$$

and the average charge per perturber

$$
\left|Z_{p} e\right|=2 \zeta e /(1+\zeta)
$$

Substituting (4) in (3) we get

$$
\log N_{p}=23.491-1.5 \log \left|Z_{p}\right|+4.5 \log Z-7.5 \log n_{m},
$$

where the logarithms are to the base 10 .

For hydrogen plasma, $Z=1$ and $\zeta=1$ and then Equation (7) becomes

$$
\log \left(N_{i}+N_{e}\right)=23.491-7.5 \log n_{m} .
$$

This is the Inglis-Teller relation. The numerical value of the first term on the righthand side is slightly different from that given by Inglis and Teller because in Equation (4) we have used the presently accepted value of the constant, namely 2.6 , while Inglis and Teller used 3.7. Nissen (1954) finds good agreement between laboratory experiments and Equation (8) with the constant term equal to 23.46. A special case of Equation (7), namely, for $\zeta=1$, has been given earlier by Underhill (1959).

We have carried out calculations, using Equation (7), for He II with $n_{m}=17$ and 21, and $\mathrm{C}$ IV with $n_{m}=15$ and 21. A series of values were used for $\zeta$. The results are shown in Table I.

TABLE I

Calculated values of the electron density for He II with $n_{m}=17$ and 21 , and C IV with $n_{m}=15$ and 21 , for a series of values of $\zeta$.

All electron density values are in the units of $10^{15} \mathrm{~cm}^{-3}$.

\begin{tabular}{lllllll}
\hline$\zeta$ & $Z_{p}$ & \multicolumn{2}{l}{ Electron density } \\
\cline { 3 - 4 } & & \multicolumn{3}{l}{ He II } & & \multicolumn{2}{l}{ C IV } \\
\cline { 3 - 4 } \cline { 5 - 6 } & & $n_{m}=17$ & $n_{m}=21$ & & $n_{m}=15$ & $n_{m}=21$ \\
\hline 1.0 & 1.0 & 2.07 & 0.42 & & 120 & 9.6 \\
1.5 & 1.2 & 1.89 & 0.39 & & 109 & 8.8 \\
2.0 & 1.333 & 1.79 & 0.37 & & 104 & 8.3 \\
2.5 & 1.429 & 1.73 & 0.36 & & 100 & 8.0 \\
3.0 & 1.5 & 1.69 & 0.35 & & 98 & 7.8 \\
3.5 & 1.556 & 1.66 & 0.34 & & 96 & 7.7 \\
4.0 & 1.6 & 1.64 & 0.34 & & 95 & 7.6 \\
\hline
\end{tabular}


It will be noticed from Table I that $N_{e}$ is relatively insensitive to $\zeta$. The uncertainties in the observational data and the approximations inherent in the theory put limitations on the degree of accuracy of the results obtained. To the degree of these limitations, the dependence on $\zeta$ can be neglected. The results obtained from He II give us an estimate of $4 \times 10^{14} \mathrm{~cm}^{-3}$ for the electron density in the emission line region of Wolf-Rayet stars. The results obtained from C IV lines lead to much higher estimates. The reasons for this discrepancy are not clear. One possibility is that it is due to stratification, the $\mathrm{C}$ IV lines being formed deeper in the atmosphere. However, Figure 7 of Smith and Aller (1971) appears to argue against this possibility. Another possibility is that the available observational data are inadequate. It may be noted that if we were to use the high-temperature approximation, the corresponding values of electron density will be all greater than those shown in Table I, except when $\zeta$ is very large $(>7)$.

We conclude that the available spectral data indicate that the electron density in the region which gives rise to He II lines in Wolf-Rayet stars is $\simeq 4 \times 10^{14} \mathrm{~cm}^{-3}$.

\section{Acknowledgement}

This work was supported in part by a research grant from the National Research Council of Canada.

\section{References}

Aller, L. H.: 1943, Astrophys. J. 97, 135.

Bappu, M. K. V.: 1973, in M. K. V. Bappu and J. Sahade (eds), 'Wolf-Rayet and High Temperature Stars', IAU Symp. 49, 59.

Bethe, H. A. and Salpeter, E. E.: 1957, Quantum Mechanics of One- and Two-Electron Atoms, Springer-Verlag, Berlin.

Edlén, B.: 1931, Ark. Mat. Astron. Fys. 22B, No. 11.

Edlén, B.: 1932, Observatory, 55, 115.

Edlén, B.: 1933, Z. Astrophys. 7, 378.

Edlén, B.: 1956, Vistas in Astronomy 2, 1456.

Griem, H. R.: 1964, Plasma Spectroscopy, McGraw-Hill Book Co., New York.

Inglis, D. R. and Teller, E.: 1939, Astrophys. J. 90, 439.

Ivanov-Kholodnyi, G. S. and Nikol'skii, G. M.: 1961, Astron. Zh. 38, 455.

Jefferies, J. T.: 1968, Spectral Line Formation, Blaisdell Publishing Co., Waltham, Mass.

Kurochka, L. N.: 1965, Astrofiz. 1, 369.

Kurochka, L. N.: 1967, Astron. Zh. 44, 368.

Kurochka, L. N.: 1974, Astron. Zh. 51, 892.

Mitrofanov, A. V.: 1973, Astron. Zh. 49, 1063.

Nissen, W.: 1954, Z. Physik 139, 638.

Smith, L. F. and Aller, L. H.: 1971, Astrophys. J. 164, 275.

Underhill, A. B.: 1959, Publ. Dom. Astrophys. Obs. 11, 209.

Underhill, A. B.: 1968, Ann. Rev. Astron. Astrophys. 6, 39.

Varshni, Y. P.: 1977, Astrophys. Space Sci. 46, 443.

Varshni, Y. P. and Lam, C. S.: 1976, Astrophys. Space Sci. 45, 87. 